\title{
Ichneumonidae (Hymenoptera) fauna of Gelincik Mountain Natural Park (Isparta, Turkey) ${ }^{1}$ \\ Gelincik Dağı Tabiat Parkı (Isparta, Turkey) Ichneumonidae (Hymenoptera) faunası Ayşegül ÖZDAN ${ }^{2 *} \quad$ Mehmet Faruk GÜRBÜZ ${ }^{2}$
}

\begin{abstract}
Summary
This study was carried out to determine the Ichneumonidae (Hymenoptera) fauna of Gelincik Mountain Natural Park. Six stations were chosen with different altitude and floristic structure and were conducted between April 2010 and October 2012. Specimens were collected via sweeping net and malaise traps. A total of 145 samples were 47 species belonging to 43 genera were identified. Of these, 14 are new records for Ichneumonidae fauna for Turkey. These species are: Arotrephes perfusor (Gravenhorst, 1829), Barichneumon peregrinator (Linnaeus, 1758), Charitopes gastricus (Holmgren, 1868), Dicaelotus pumilus (Gravenhorst, 1829), Diphyus trifasciatus (Gravenhorst, 1829), Gelis gallicator (Aubert, 1971), Herpestomus arridens (Gravenhorst, 1829), Ichneumon caloscelis Wesmael, 1845, Lissonota proxima Fonscolombe, 1854, Mesoleius melanoleucus (Gravenhorst, 1829), Odontocolon quercinus (Thomson, 1877), Olesicampe fulcrans (Thomson, 1887), Symplecis invisitata Rossem, 1981 and Tropistes falcatus (Thomson, 1884). Also, three genera, Arotrephes, Charitopes and Tropistes are new records for Turkey.
\end{abstract}

Keywords: Fauna, Gelincik Mountain Natural Park, Hymenoptera, Ichneumonidae, new records

\section{Özet}

Bu çalışma Gelincik Dağı Tabiat Parkı Ichneumonidae faunasını araştırmak üzere gerçekleştirilmiştir. Farklı yükseklik ve floristik yapıya sahip altı istasyon seçilmiş ve çalışmalar Nisan 2010-Ekim 2012 tarihleri arasında yapılmıştır. Örnekler atrap ve malaise tuzağı ile toplanmıştır. Toplam 145 birey toplanmıştır ve 43 cinse bağlı 47 tür teşhis edilmiştir. Bu türler arasından 14 tür Türkiye Ichneumonidae faunası için yeni kayıttır. Bu türler: Arotrephes perfusor (Gravenhorst, 1829), Barichneumon peregrinator (Linnaeus, 1758), Charitopes gastricus (Holmgren, 1868), Dicaelotus pumilus (Gravenhorst, 1829), Diphyus trifasciatus (Gravenhorst, 1829), Gelis gallicator (Aubert, 1971), Herpestomus arridens (Gravenhorst, 1829), Ichneumon caloscelis Wesmael, 1845, Lissonota proxima Fonscolombe, 1854, Mesoleius melanoleucus (Gravenhorst, 1829), Odontocolon quercinus (Thomson, 1877), Olesicampe fulcrans (Thomson, 1887), Symplecis invisitata Rossem, 1981 ve Tropistes falcatus (Thomson, 1884). Arotrephes, Charitopes ve Tropistes cinsleri de Türkiye için yeni kayıttır.

Anahtar sözcükler: Fauna, Gelincik Dağı Tabiat Parkı, Hymenoptera, Ichneumonidae, yeni kayıtlar

\footnotetext{
${ }^{1}$ This study was supported by the Scientific and Technological Research Council of Turkey (TUBiTAK TOVAG Project No:1110538). Financial support was SDÜ BAP project No. 2273-D-10.

${ }^{2}$ Süleyman Demirel University, Faculty of Arts and Sciences, Department of Biology, 32260, Isparta, Turkey

* Corresponding author (Sorumlu yazar) e-mail: aysegulozdan@gmail.com

Received (Alınış): 28.05.2016 Accepted (Kabul ediliş): 27.10.2016 Published Online (Çevrimiçi Yayın Tarihi): 13.12 .2016
} 


\section{Introduction}

Ichneumonidae is the largest hymenopteran family with 51 generally recognized subfamilies, 1579 genera and 24281 described species globally (Yu et al., 2012). The estimated total number of species varies from 60000 (Townes, 1969; Wahl \& Sharkey, 1993) to more than 100000 . Ichneumonids are distributed worldwide and play a significant role in natural and agricultural ecosystems as parasitoids of arthropods (Kopylov \& Zhang, 2015).

Ichneumonids have been used successfully as biocontrol agents and given the largely undocumented fauna there is huge potential for their utilization in managed biocontrol programs (Ghahari et al., 2012).

Faunistic research on the family Ichneumonidae in Turkey started in the 19th century (Çoruh et al., 2016). In the catalog of Kolarov (1995) only 383 species are listed. The last decade new records have been added to this fauna. With this study, the number of Ichneumonidae species in Turkey increased to 1234.

Gelincik Mountain National Park is located in Isparta, Turkey, has a great potential in recreational terms with its rich natural and cultural resources. This area has a rich flora important both for its biodiversity as well as for including many ornamental, medicinal and aromatic plants with $24.6 \%$ endemism. Similarly, the area is important for reproduction, nutrition and survival for different animal species (Erduran \& Cırık, 2011). To further our knowledge of this valuable natural resource, the aim of this study was to survey the Ichneumonidae fauna of Gelincik Mountain Natural Park.

\section{Material and Methods}

\section{Sampling}

Ichneumonidae samples were collected from April to October between 2010 and 2012. Six stations which have different altitude and floristic structure were chosen in Gelincik Mountain Natural Park in Isparta Province (Figure 1).

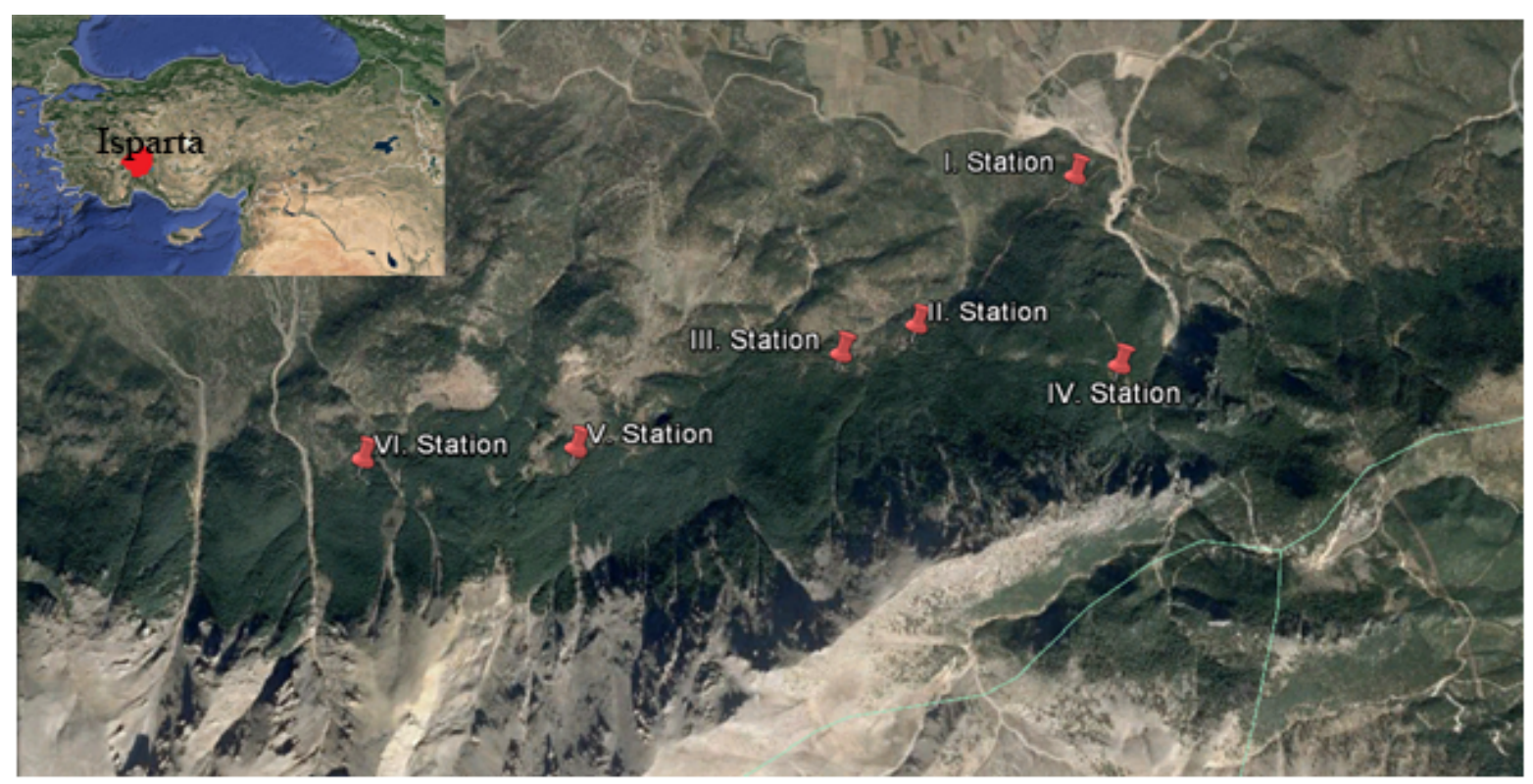

Figure 1. Sampling sites (stations) in Gelincik Mountain Natural Park.

Station I ( $38^{\circ} 06.584^{\prime} \mathrm{N} 30^{\circ} 44.837^{\prime} \mathrm{E}$; $\left.1100-1150 \mathrm{~m}\right)$ : The vegetation is characterized by Quercus coccifera. Other plant species commonly encountered in the area are Achillea sp. L., Aegilops geniculata Roth., Centaurea iberica Trev. Ex Sprengel., Juniperus oxycedrus L., Lamium maculatum L. and Trifolium repens $\mathrm{L}$. 
Station II (38 $\left.06.020^{\prime} \mathrm{N} 30^{\circ} 44.112^{\prime} \mathrm{E} ; 1250-1300 \mathrm{~m}\right)$ : The vegetation consists of Cedrus libani A. Rich, Lepidium draba L., Centaurea depressa M. Bieberstein, Convolvulus arvensis L., Crataegus orientalis Pallas ex Bieb., Geranium tuberosum L., Pinus nigra Arnold, Ranunculus ficaria L., Ranunculus cuneatus Boiss. and Trifolium davisii Hossain.

Station III (3805.916' N 30 43.776' E; 1300-1350 m): Cedrus libani A. Rich, Pinus nigra Arnold, and Poa sp. L. are dominant plants in the area. Other notable plant species are Cirsium sp. Miller, Crataegus orientalis Pallas ex Bieb., Malva sp. L., Scutellaria sp. L., Sideritis sp. L., Stachys cretica L. and Vicia sp. L.

Station IV (38 $05.869^{\prime} \mathrm{N} 30^{\circ} 44.985^{\prime} \mathrm{E}$; $\left.1450-1500 \mathrm{~m}\right)$ : The vegetation is characterized by Cedrus libani A. Rich, Crataegus orientalis Pallas ex Bieb., Jasminum sp. L. Pinus nigra Arnold, Rosa dumalis Bechst. and Stachys cretica L.

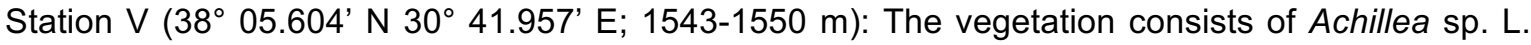
Centaurea sp. L., Centaurea iberica L., Cotoneaster sp. Medik., Potentilla sp. L. and Sedum sp. L.

Station VI (38 5,488' N 30 41,422 E; 1520 m): The vegetation is characterized by Cedrus libani A. Rich. and Pinus nigra Arnold.

\section{Sampling and collection}

Ichneumonidae specimens were sampled using malaise traps and sweep net. Samples were taken from malaise traps about every fifteen days. Ichneumonid species were separated from the other insects under a stereomicroscope in the laboratory. The samples collected by sweep net were taken into $70 \%$ ethanol solution in the plastic containers. Collected ichneumonids were taken to the laboratory to be mounted and labeled. All of the samples were lodged in the collection of the Department of Biology, Faculty of Arts and Sciences at Süleyman Demirel University, Isparta, Turkey.

\section{Results and Discussion}

Forty-seven species belonging to 43 genera were identified with 14 of these new records for Turkey. With this new records, the number of Ichneumonidae species in Turkey increased to 1234.

A list of the species is given below along with the collection date, locations, specimen numbers of each sex and distribution in Turkey. For newly recorded species, hosts, associated plants, general distributions are also given according to Yu et al., 2012. Newly recorded species are indicated with an asterisk.

\section{List of the species}

\section{Subfamily Anomaloninae}

\section{Anomalon cruentatum Geoffroy, 1785}

Material examined: GDTP, II. Station, 3.VII.2010, q, I. Station, 3.VII.2010, + , III. Station, 29.V.2010, ㅇ, VI. Station, 17.VI.2012, 2 우, 24.VI.2012, V. Station, + .

Distribution in Turkey: Afyon, Muğla (Kolarov et al., 2002) Isparta-Merkez-Kirazlıdere-Gönen (Gürbüz, 2004); Antalya, Bayburt, Bingöl, Diyarbakır, Erzincan, Erzurum, Iğdır, Kahramanmaraş, Kars (Çoruh et al., 2004), Adıyaman-Merkez, Batman-Hasankeyf, Diyarbakır-Çermik-Merkez, Elazığ- Yemişlik, Malatya-Yeşilyurt-Doğanyurt, Mardin-Savur (Akkaya, 2005), Isparta-Gölcük-Çünür (Buncukçu, 2008), Isparta-Kasnak Meşesi Tabiatı Koruma Alanı (Kırtay, 2008), Isparta-Davraz (Birol, 2010), Erzurum, Tunceli (Kolarov et al., 2014a), Bayburt, Kars, Erzurum (Çoruh \& Kolarov, 2016).

\section{Barylypa uniguttata (Gravenhorst, 1829)}

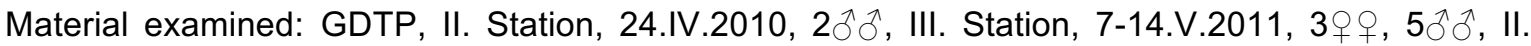
Station, 16-30.V.2011, 2우․ 2 ${ }^{\lambda}{ }^{\lambda}$, IV. Station, 17.VI.2012, 우. 
Distribution in Turkey: Çanakkale (Kolarov et al., 1994), Isparta (Gürbüz et al., 2009a).

\section{Subfamily Banchinae}

\section{Lissonota (Loxonota) histrio (Fabricius, 1798)}

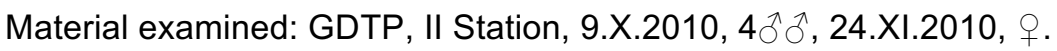

Distribution in Turkey: Erzurum (Pekel \& Özbek, 2000); Diyarbakır-Merkez, Elazığ-Hazar, MardinSavur (Akkaya, 2005), Ordu (Kolarov et al., 2016).

\section{*Lissonota (Lissonota) proxima Fonscolombe, 1854}

Material examined: GDTP, II. Station, 9.X.2010, ̂, III. Station, 15.X.2012, ㅇ.

Diagnosis: First flagellomere segment four times as long as wide (Figure 2b). Mesosoma black. Legs red, trochanters black. Tarsal claws not pectinate (Figure 2c). Tegulae brown. Fore wing with areolet (Figure 2d). Radial vein (R3) beyond areolet straight or weakly curved. Second and third metasomal tergites completely reddish and punctated. Propodeum and hind coxae densely punctated (Figure 2e). Metasoma with red pattern (Figure 2a). Coxa, femur, tibia red.

Associated with plant: Angelica sylvestris L. (Apiales: Apiaceae), Chaerophyllum aromaticum L. (Apiales: Apiaceae).

General Distribution: Palearctic.

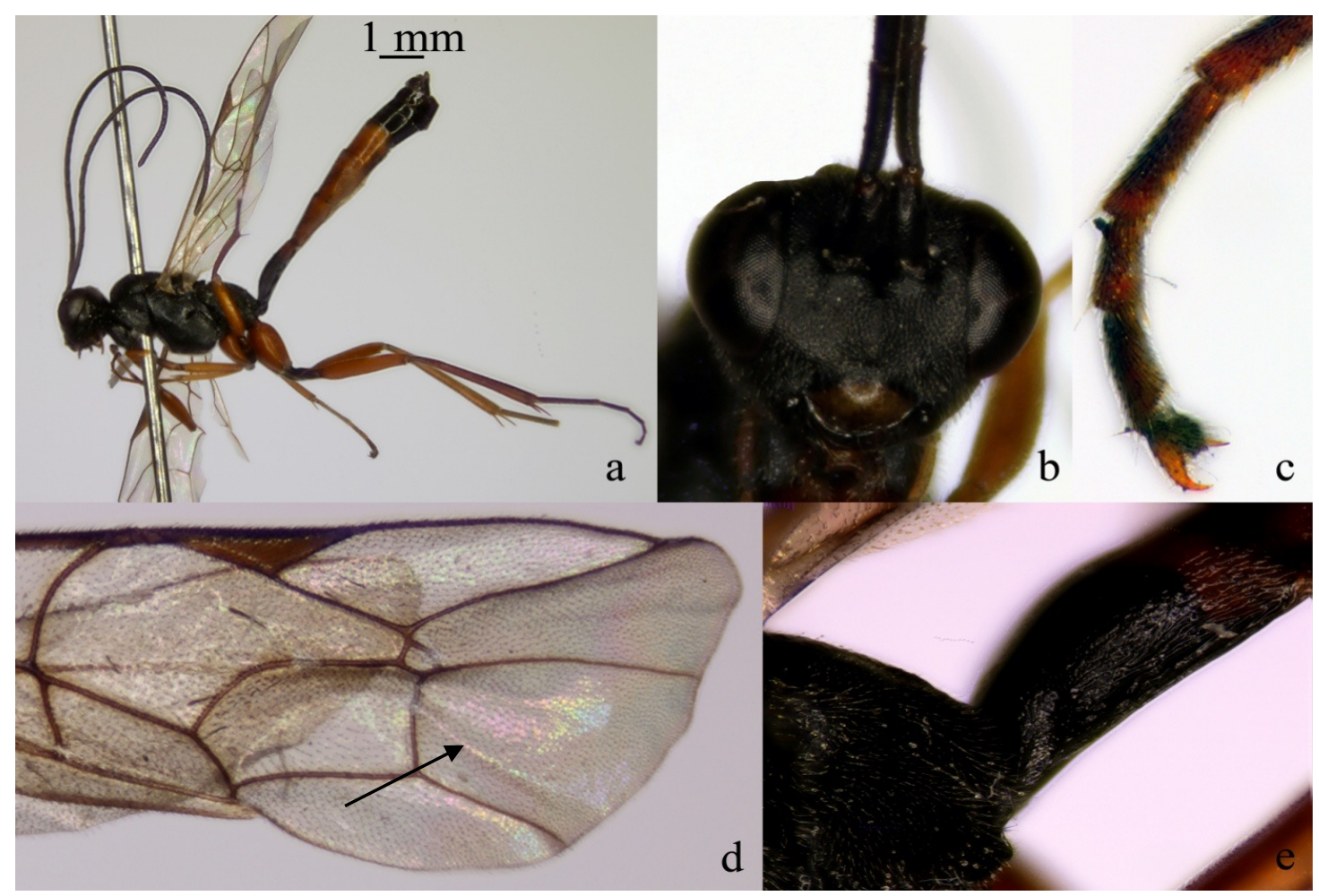

Figure 2. Lissonota (Lissonota) proxima a. habitus, lateral view, b. head, facial view, c. tarsus and tarsal claws, d. forewing with areolet and e. propodeum, first metasomal tergite.

\section{Subfamily Campopleginae}

\section{Campoletis viennensis (Gravenhorst, 1829)}

Material examined: GDTP, III. Station, 3.VII.2010, q. 
Distribution in Turkey: Adana (Kolarov \& Beyaslan, 1995), Bayburt (Özbek et al., 2000, Çoruh et al., 2014b), Hatay (Çoruh et al., 2013), Bayburt (Çoruh \& Kolarov, 2016).

\section{Cymodusa australis (Smits van Burgst, 1913)}

Material examined: GDTP, II. Station, 27.V.2012, ㅇ.

Distribution in Turkey: Edirne (Kolarov \& Beyarslan, 1995).

\section{*Olesicampe fulcrans (Thomson, 1887)}

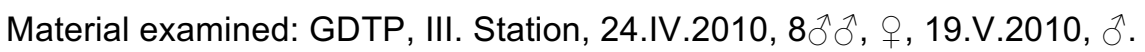

Diagnosis: Eyes with inner margins not converging strongly ventrally (Figure 3b). Temple long and swollen. The lower tooth of mandible often longer than the upper. Base of mandible and scape yellow. Fore wing with areolet. Propodeal spiracles circular or elongate (Figure 3c). Metatrochanter conspicuous (Figure 3d). Metasomal tergites III-V red, femur and tibia red (Figure 3a).

General Distribution: Palearctic.

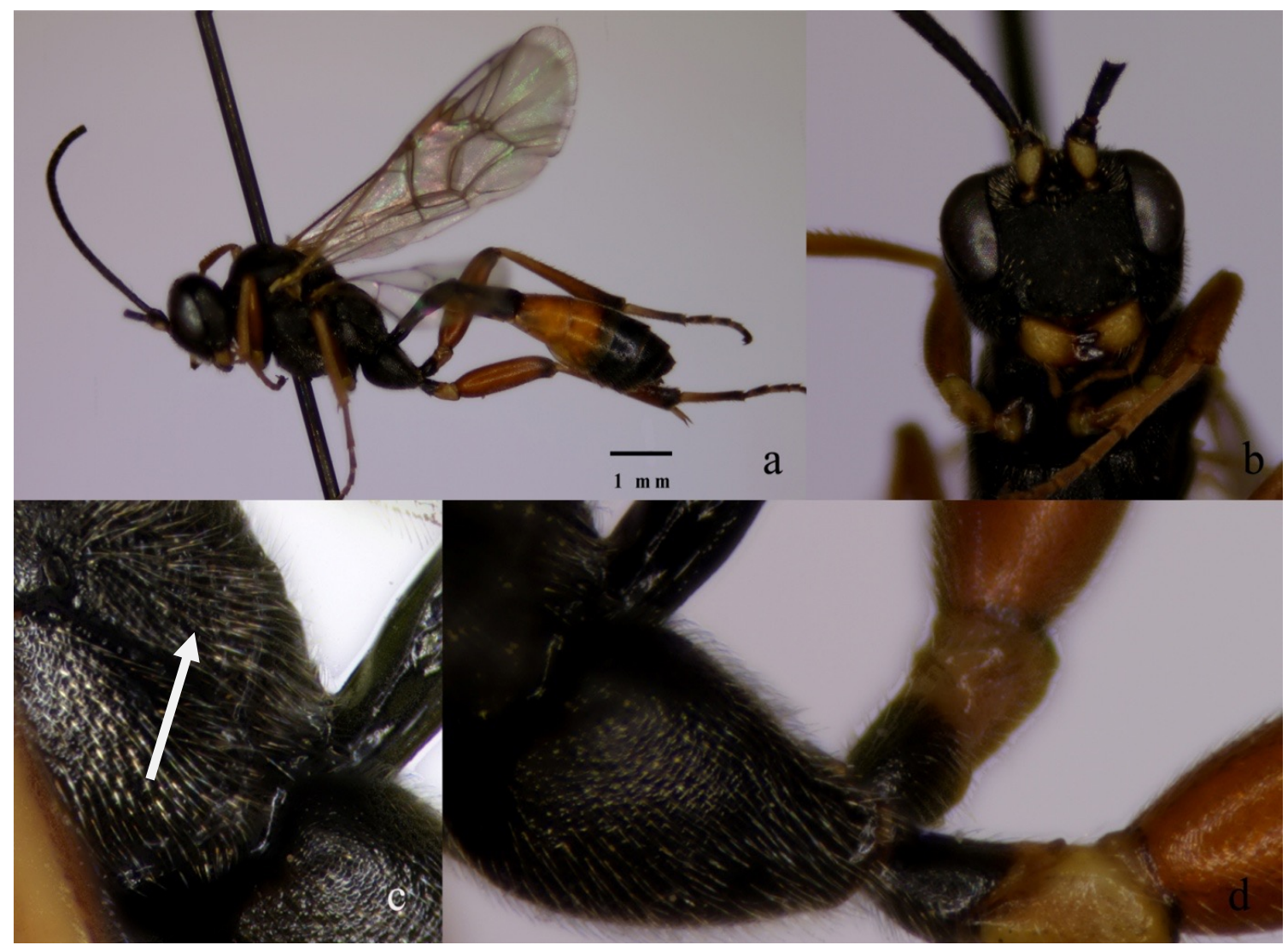

Figure 3. Olesicampe fulcrans a. habitus, lateral view, b. head, facial view, c. propodeum and first tergite and d. metatrochanter, lateral view.

\section{Subfamily Cremastinae}

Pristomerus pallidus (Kriechbaumer, 1884)

Material examined: GDTP, I. Station, 27.V.2012, q.

Distribution in Turkey: Erzurum (Pekel \& Özbek, 2000). 


\section{Subfamily Cryptinae}

\section{Aritranis director (Thunberg, 1824)}

Material examined: GDTP, II. Station , 29.V.2011, đิ, GDTP, III. Station, 19. V.2010, ô, 29.V.2010,

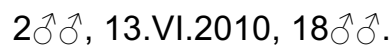

Distribution in Turkey: Isparta, Egirdir, Yalvaç, Burdur, Antalya (Gürbüz \& Kolarov, 2008); Kasnak Meşesi Tabiatı Koruma Alanı-Isparta (Gürbüz et al., 2009b).

\section{Buathra tarsoleucos (Schrank, 1781)}

Material examined: GDTP, II. Station, 12.VIII.2012, ․ .

Distribution in Turkey: Isparta (Gürbüz \& Kolarov, 2008).

\section{Cryptus tuberculatus Gravenhorst, 1829}

Material examined: GDTP, III. Station, 29.V.2010 क, 13.VI.2010, ㅇ, I. Station, 13.VI.2010, §ิ.

Distribution in Turkey: Locality not given (Sevidy, 1959), Tekirdağ (Kolarov \& Yurtcan, 2008).

\section{Cryptus viduatorius Fabricius, 1804}

Material examined: GDTP, III. Station, 24.IV.2010, 2우, 3.VII.2010, ㅇ.

Distribution in Turkey: İstanbul (Kolarov, 1995); Isparta, Egirdir (Gürbüz \& Kolarov, 2008); Kasnak Meşesi Tabiatı Koruma Alanı-Isparta (Gürbüz et al., 2009b), Erzurum (Çoruh \& Çoruh, 2008; Çoruh et al., 2014b), Rize (Çoruh et al., 2014a), Erzurum (Çoruh \& Kolarov, 2016; Çoruh et al., 2016).

\section{Myrmeleonostenus italicus (Gravenhorst, 1829)}

Material examined: GDTP, III. Station, 29.V.2010, $q$.

Distribution in Turkey: Isparta, Antalya (Gürbüz \& Kolarov, 2008); Kasnak Meşesi Tabiatı Koruma Alanı-Isparta (Gürbüz et al., 2009b); Erzincan (Çoruh et al., 2016).

\section{Stenarella domator (Poda, 1761)}

Material examined: GDTP, III. Station, 31.VII.2010, ㅇ, II. Station, 12.VII.2012, ô.

Distribution in Turkey: Belgrat Ormanları (Kolarov, 1995); Isparta, Egirdir (Gürbüz \& Kolarov, 2008); Kasnak Meşesi Tabiatı Koruma Alanı-Isparta (Gürbüz et al., 2009b).

\section{Xylophrurus apum (Thomson, 1873)}

Material examined: GDTP, II. Station, 13.VI.2010, o.

Distribution in Turkey: Afyon-Sultan Dağı (Özdemir \& Güler, 2009).

\section{* Arotrephes perfusor (Gravenhorst, 1829)}

Material examined: GDTP, II. Station, 1.VII.2012, o.

Diagnosis: Face shiny, Clypeus convex and black (Figure 4d). Antenna reddish brown. Legs red (Figure 4a). Notaulus not reaching to center of mesoscutum (Figure 4b). Tegula brownish. Wings normal. Areolet present. Second intercubitus $(3 \mathrm{rs}-\mathrm{m})$ distinct. Second recurrent vein $(2 \mathrm{~m}-\mathrm{Cu})$ with two bullae (Figure 4c). Postpectal carina incomplete. Center of pronotum without carina. Second and third metasomal tergites black. Body black.

General Distribution: Western Palearctic. 


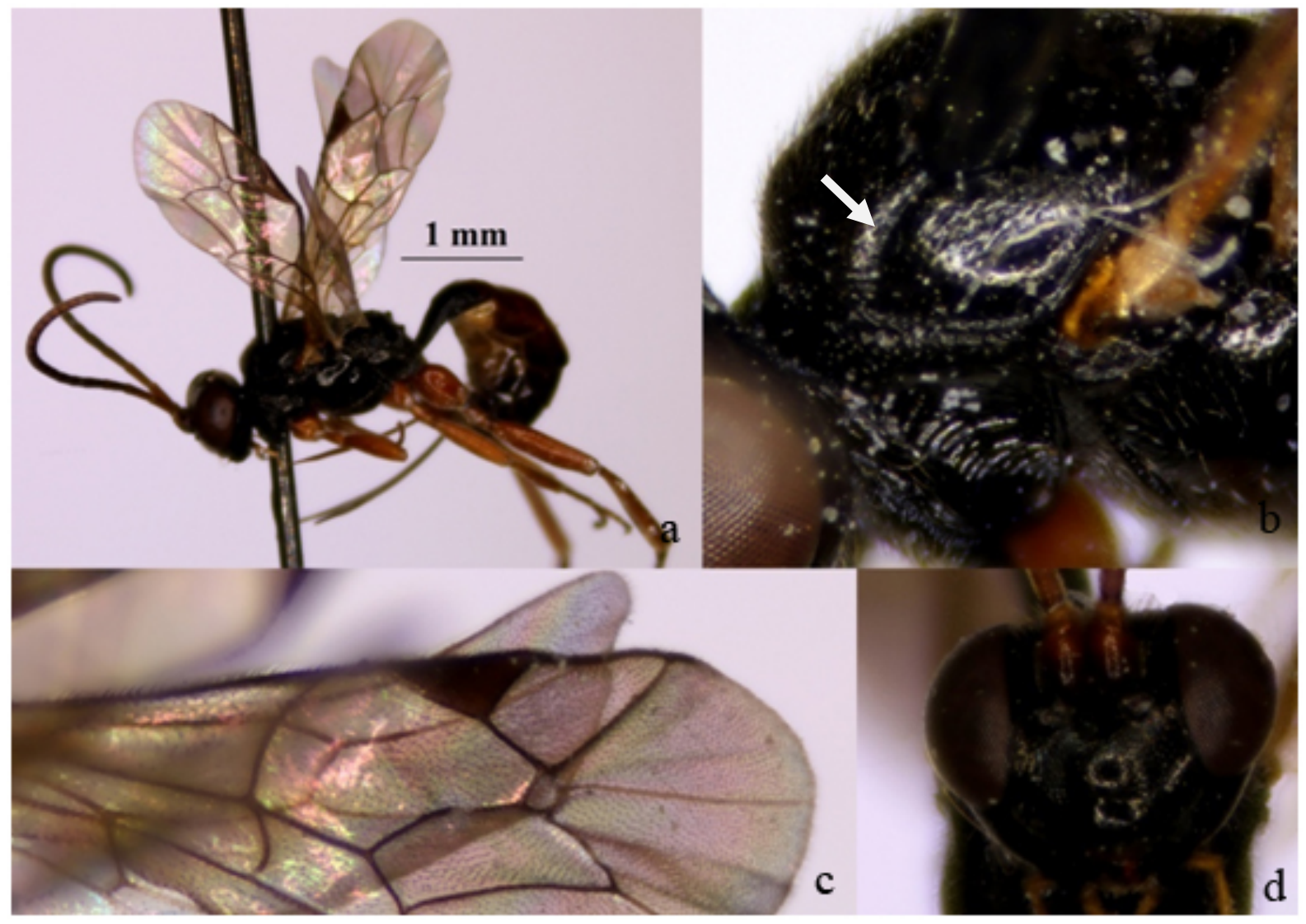

Figure 4. Arotrephes perfusor a. habitus, lateral view, b. view of Notaulus, c. forewing and d. head, facial view.

\section{${ }^{*}$ Charitopes gastricus (Holmgren, 1868)}

Material examined: GDTP, III. Station, 14.V.2011, +.

Diagnosis: Eyes without hairs. Antenna red-brown. Fifth flagellomere 1.7-1.9 times as long as wide (Figure $5 \mathrm{~b})$. 2. recurrent vein (2m-cu) complete, inclivous and with two bullae (Figure $5 \mathrm{c}$ ). Body prolonged (Figure 5a). Mesoscutum mat. Tergites polished, red-brown (Figure 5d). Ovipositor sheath about 0.350.65 times as long as forewing. Ovipositor sheath about 1.1 times as long as metatibia.

General Distribution: Palearctic, Nearctic, Neotropical.

\section{Gelis areator (Panzer, 1804)}

Material examined: GDTP, II. Station, 17.VI.2012, 2 웅.

Distribution in Turkey: Edirne (Okyar et al., 2012).

\section{* Gelis gallicator (Aubert, 1971)}

Material examined: GDTP, II. Station, 1.VII.2012, ㅇ.

Diagnosis: Head, antenna red (Figure 6b). Clypeus convex. Mesosoma red. Epomia short or absent. Mesosoma mat (Figure 6c). Areolet absent. Pterostigma brown. Fore wing with single brown band (Figure 6d). Ovipositor sheath shorter than metatibia (Figure 6a).

General Distribution: Western Palearctic. 


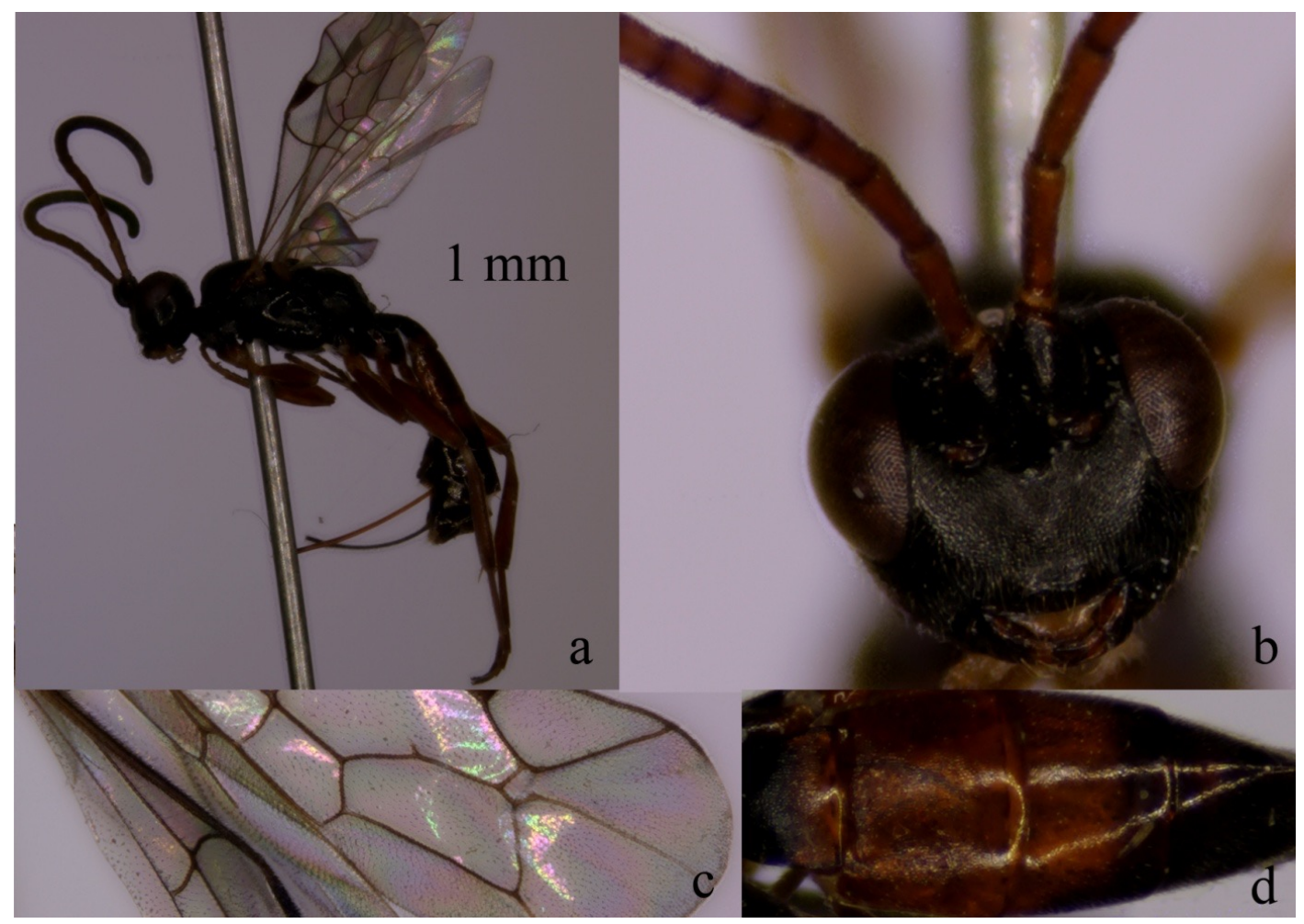

Figure 5. Charitopes gastricus a. habitus, lateral view, b. head, facial view, c. forewing and d. metasoma dorsal view.

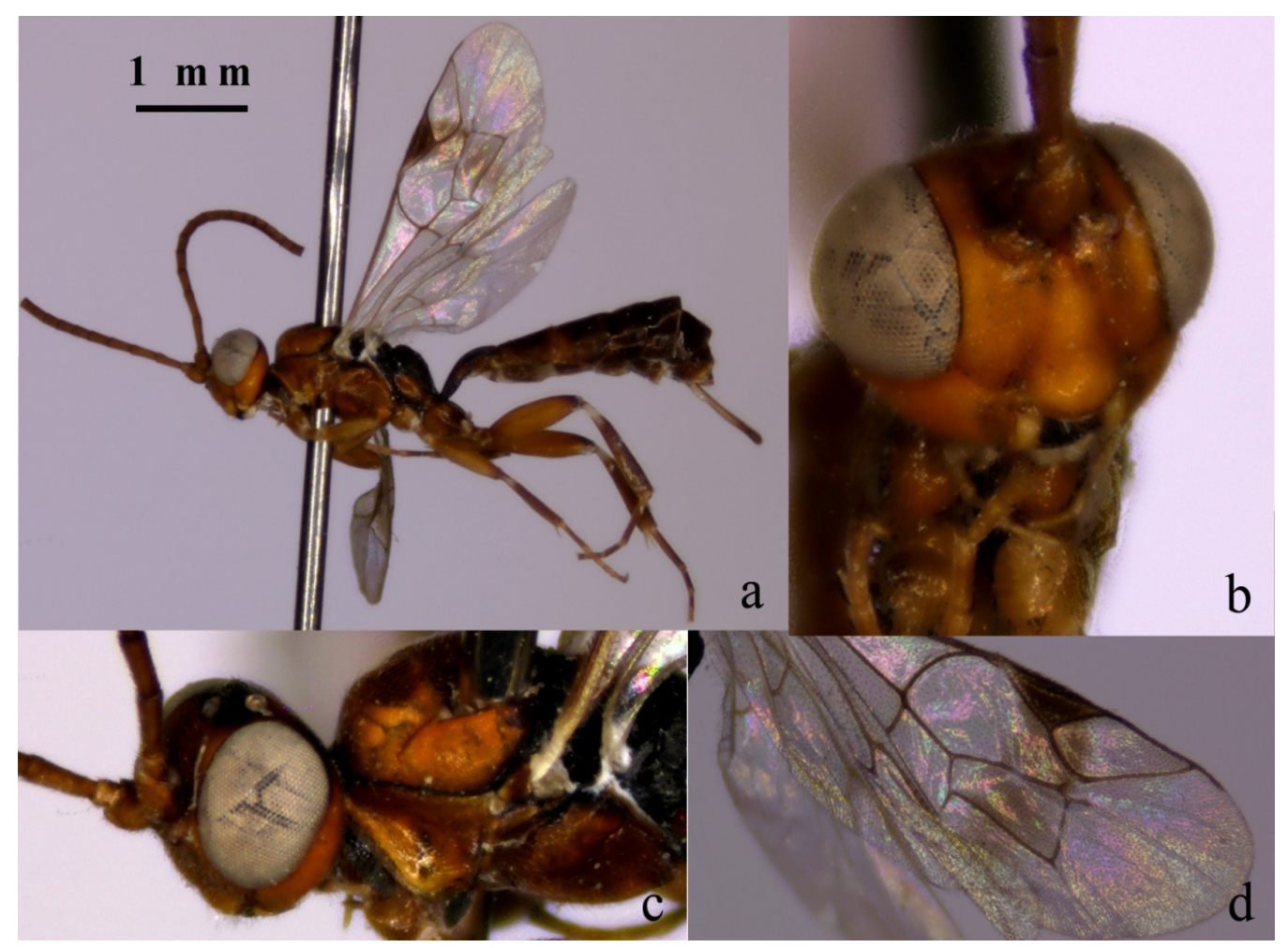

Figure 6. Gelis gallicator a. habitus, lateral view, b. head, facial view, c. head and mesosoma, lateral view and d. forewing. 


\section{*Tropistes falcatus (Thomson, 1884)}

Material examined: GDTP, II. Station, 1.VII.2012, ㅇ.

Diagnosis: Clypeus and mandible black (Figure 7b). Mesosoma laterally compressed (Figure 7a). Wings fully complete. Areolet present. Vein 3rs-m incomplete or little complete. Nervellus slightly vertical or inclivous (Figure 7c). Notaulus not reaching to center of mesoscutum (Figure 7d). Postpectal carina incomplete. Second epipleural tergite separated with fold. Legs red. Second metasomal tergite red and with black punctated. Base of third metasomal tergite red.

General Distribution: Palearctic.

\section{Subfamily Ctenopelmatinae}

\section{*Mesoleius melanoleucus (Gravenhorst, 1829)}

Material examined: GDTP, III. Station, 13.VI.2010, + .

Diagnosis: Face black, Clypeus and mandible yellow (Figure 8b). Lower tooth of mandible not longer than upper tooth. Flagellum red. Antennae with 38-45 flagellomeres. Prepectal carina reaching to anterior mesopleuron. Mesopleuron distinctly punctated (Figure 8c). Areolet absent. Nervellus intercepted about at its middle or below middle. Legs yellow (Figure 8a). Tarsal claws not pectinate. Glymma present. Epipleura of II and III metasomal segments separate with ledge. Second metasomal tergite without longitudinal carina. Ovipositor with subapical dorsal notch.

General Distribution: Western Palearctic

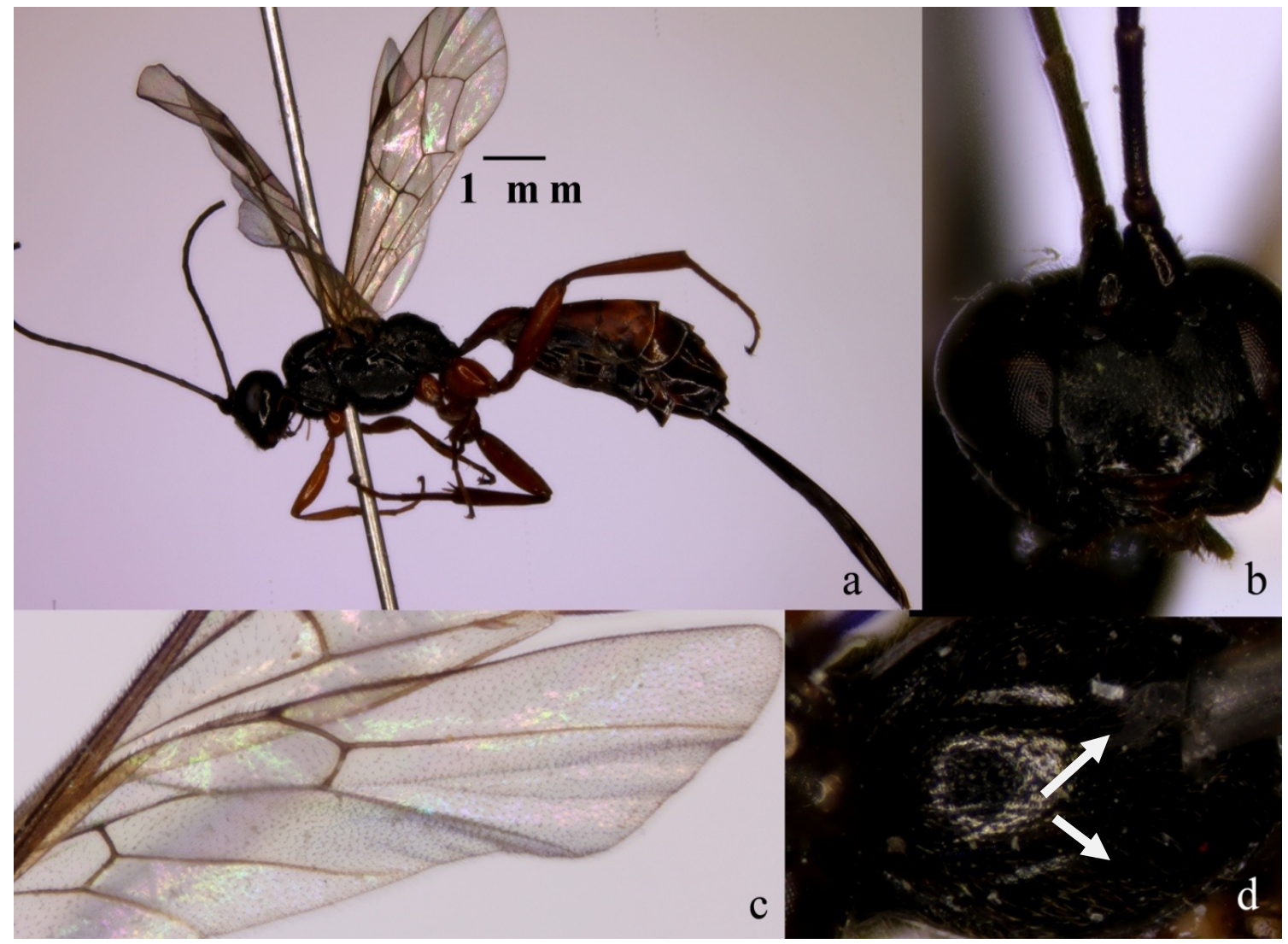

Figure 7. Tropistes falcatus a. habitus, lateral view, b. head, facial view, c. hindwing and d. view of notaulus. 


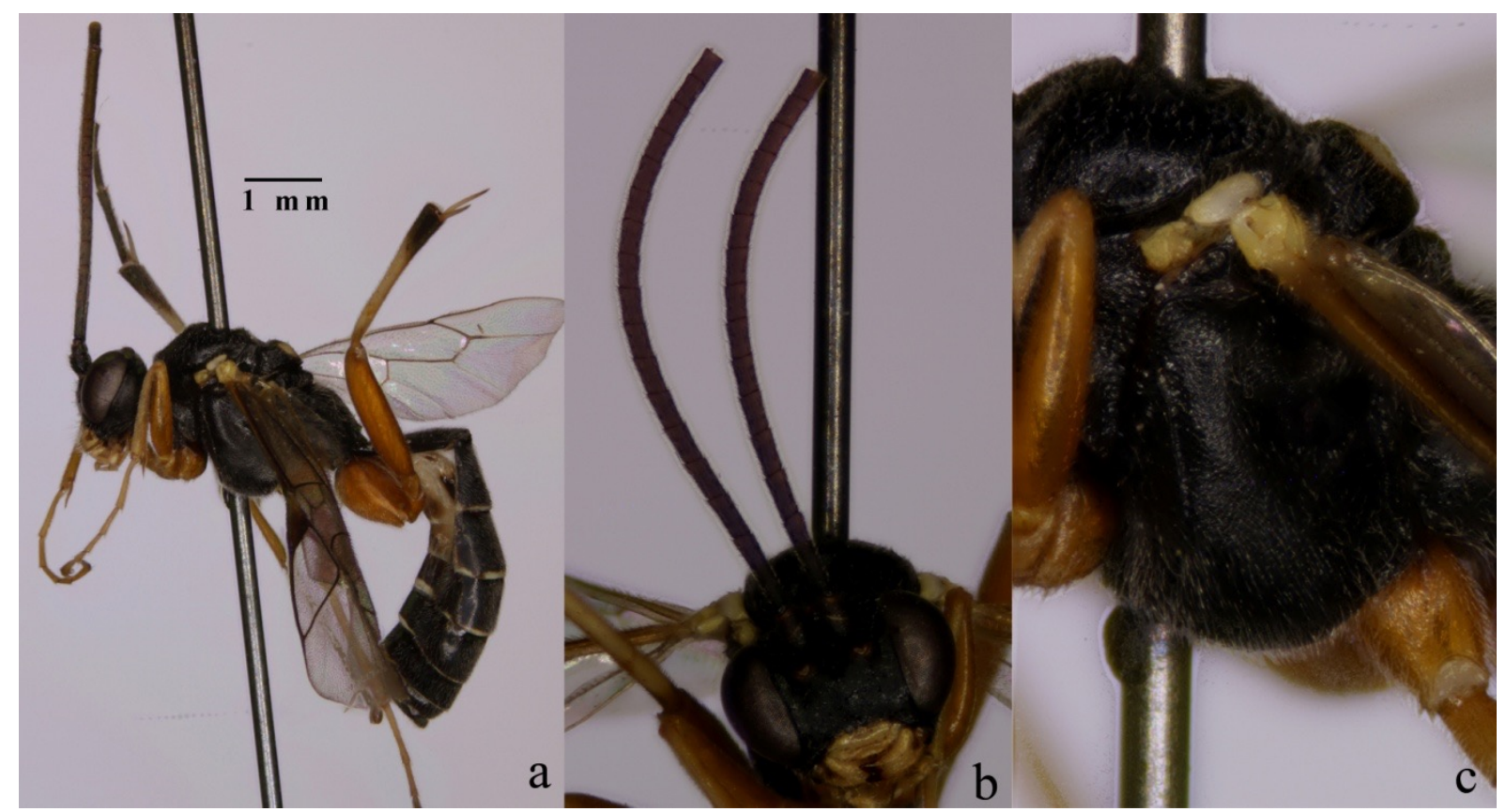

Figure 8. Mesoleius melanoleucus a. habitus, lateral view , b. head, facial view and c. mesopleuron, lateral view.

\section{Subfamily Diplazontinae}

\section{Diplazon tibiatorius (Thunberg, 1822)}

Material examined: GDTP, III. Station, 19.V.2010, ․

Distribution in Turkey: Ankara (Kolarov, 1995); Ankara (Özdemir, 2001); Tekirdağ (Yurtcan et al., 1999).

\section{Subfamily Ichneumoninae}

\section{Anisobas cingulatellus Horstmann, 1997}

Material examined: GDTP, II. Station, 25.IX.2010, q.

Distribution in Turkey: Edirne, Tekirdağ (Yurtcan et al., 1999); Erzurum (Riedel et al., 2010).

\section{Colpognathus celerator (Gravenhorst, 1807)}

Material examined: GDTP, 1.VII.2012, VI. Station, $\hat{\jmath}$.

Distribution in Turkey: Erzurum (Çoruh \& Özbek, 2008), Giresun, Ordu, Trabzon (Kolarov et al., 2014b), Giresun (Çoruh et al., 2016).

\section{*Dicaelotus pumilus (Gravenhorst, 1829)}

Material examined: GDTP, III. Station, 19.V.2010, ô.

Diagnosis: Head black, not bulbous, frons slightly convex, with distinct punctuation, clypeus clearly separate from face, upper mandibular tooth longer than lower tooth. Lower tooth of mandible strong (Figure 9b). Antennae reddish brown. Scape black. Postanellus shorter than second flagellomere. Frontal orbit not yellow. Metasoma partly black with red pattern (Figure 9a). Pterostigma brown. Second and third metasomal tergites punctated (Figure 9c). Thyridia absent.

General Distribution: Palearctic. 


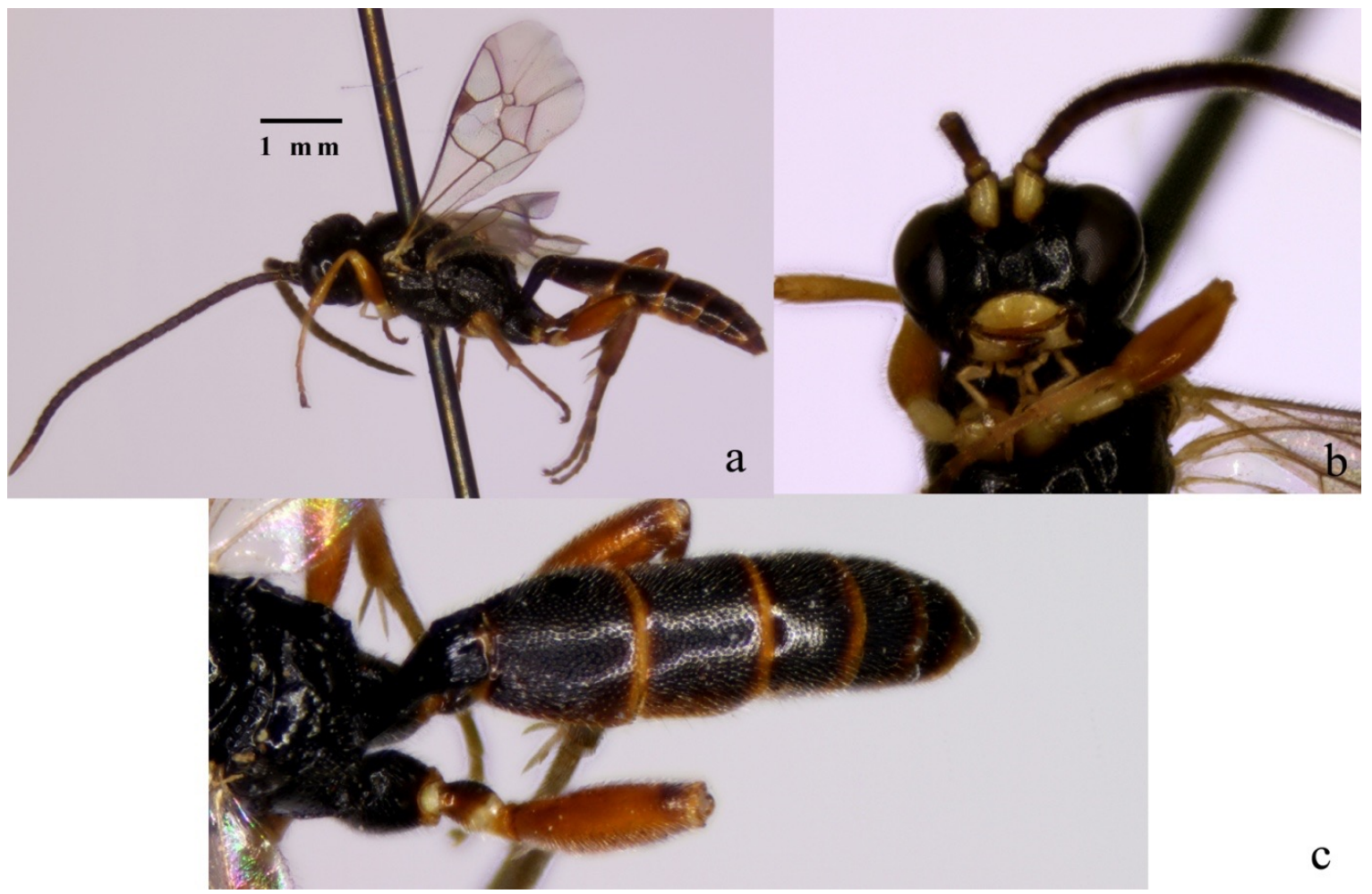

Figure 9. Dicaelotus pumilus a.habitus, lateral view, b. head, facial view and c. metasoma, dorsolateral view.

\section{${ }^{*}$ Herpestomus arridens (Gravenhorst, 1829)}

Material examined: GDTP, II. Station, 29.V.2010, q, V. Station, 2.VII.2012, q, II. Station, q.

Diagnosis: Face, Clypeus and frontal orbit red (Figure 10b). Antenna red. Mesosoma laterally compressed. Corner of pronotum and Tegula yellow (Figure 10c). Legs red. Vein Cu antefurcal (Figure 10a). Postpetiol scarce punctated or female straight (Figure 10d).

General Distribution: Palearctic.

\section{Diadromus albinotatus (Gravenhorst, 1829)}

Material examined: GDTP, III. Station, 5.XI.2011, $q$.

\section{*Diphyus trifasciatus (Gravenhorst, 1829)}

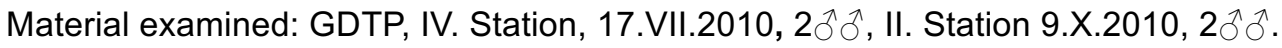

Diagnosis: Lower tooth of mandible shorter than upper tooth and different color (Figure 11b). Third and fourth flagellomeres prolonged. Facial orbit large and yellow. Metasoma densely yellow (Figure 11c). Corner of pronotum and Tegula yellow. Mesoscutum weakly punctate. Scutellum quite large (Figure 11d). Meta tibia yellow (Figure 11a). Seventh of metasomal tergit quite short.

General Distribution: Palearctic. 


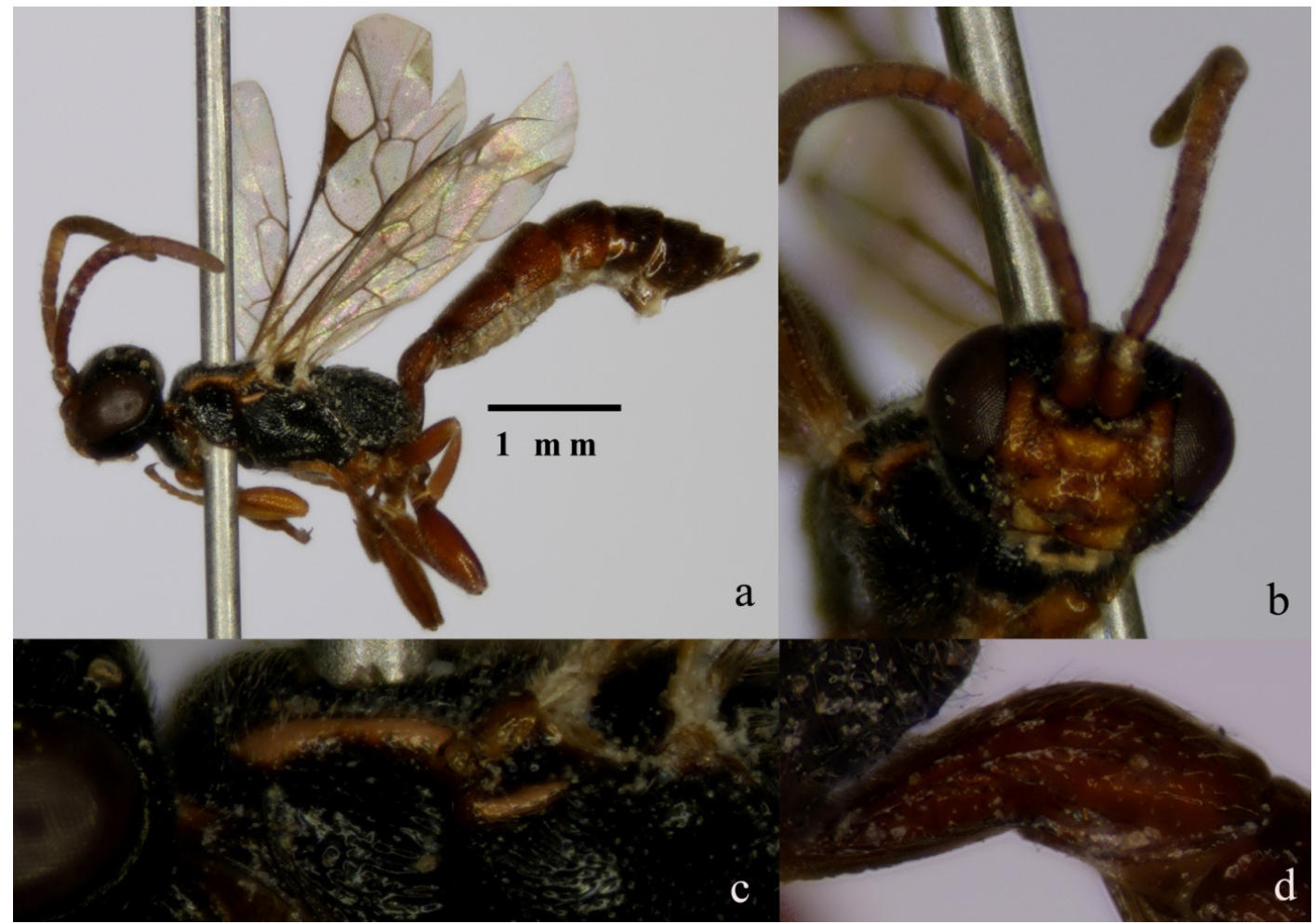

Figure 10. Herpestomus arridens a. habitus, lateral view, b. head, facial view, c. pronotum, and mesopleuron, lateral view and d. first tergite of metasoma.

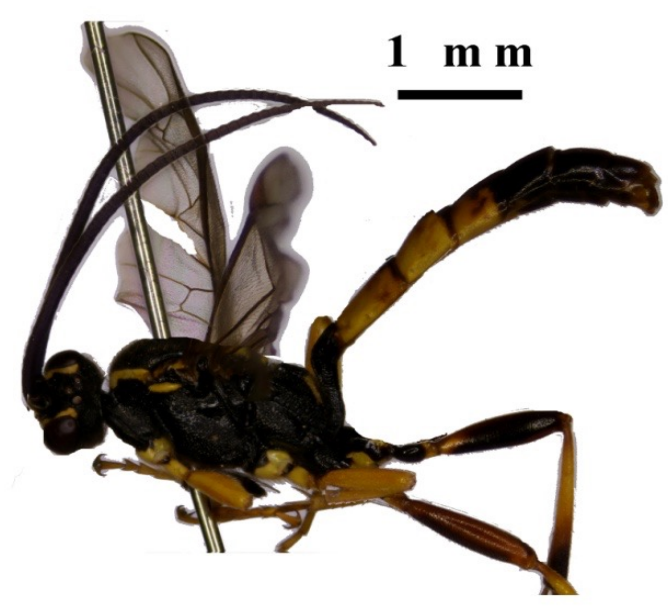

$\mathrm{a}$

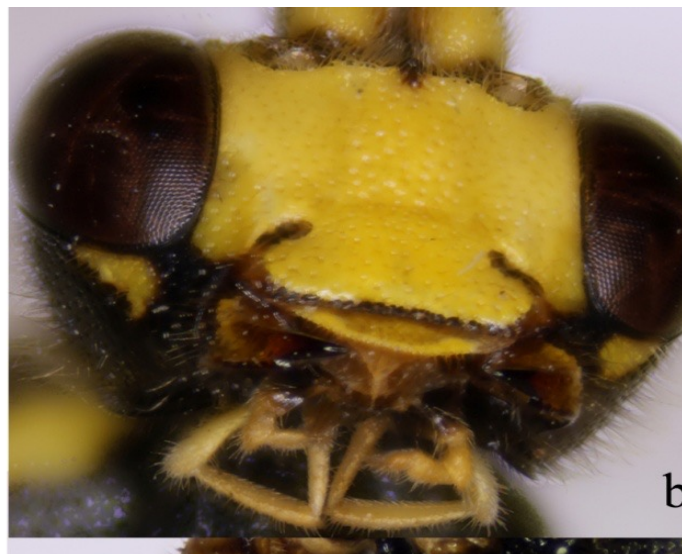

b

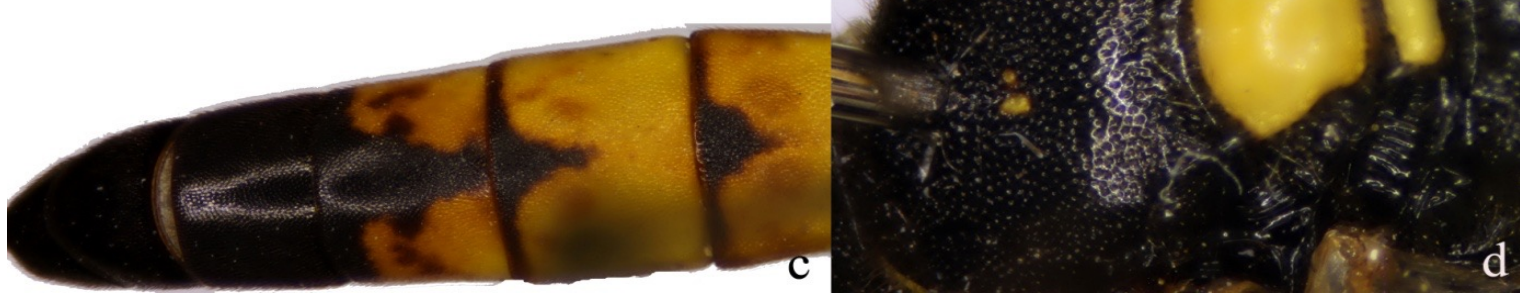

Figure 11. Diphyus trifasciatus a. habitus, lateral view, b. head, facial view, c. metasoma, dorsal view and d. mesoscutum and scutellum, dorsal view. 


\section{*Ichneumon caloscelis Wesmael, 1845}

Material examined: GDTP, II. Station, 9.X.2010, $2 \widehat{\partial} \sigma^{\lambda}$.

Diagnosis: Lower tooth of mandible distinct (Figure 12b). Antenna with 32 flagellomeres. First flagellomere segment elongate. Temple behind eyes narrowed. Propodeum without tooth, densely punctated (Figure 12c). Scutellum yellow, mesoscutum almost black (Figure 12d). Area supermedia transverse or square. Metatibia yellow with black apex (Figure 12a).

General Distribution: Palearctic.

\section{Spilichneumon occisorius (Fabricius, 1793)}

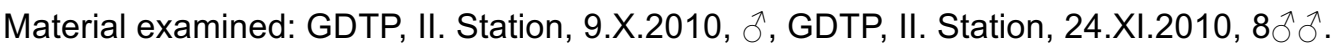

Distribution in Turkey: Konya, Eskişehir (Özdemir, 1996).

\section{Virgichneumon digrammus (Gravenhorst, 1820)}

Material examined: GDTP, IV. Station, 17.VII.2010, ㅇ, 16.VII.2011, ㅅ, ㅇ.

Distribution in Turkey: Erzurum (Riedel et al., 2010).

\section{*Barichneumon peregrinator (Linnaeus, 1758)}

Material examined: GDTP, III. Station, 5.XI.2011, + .

Diagnosis: Face black, Frontal orbit white (Figure 13b). Flagellomeres small, fifth flagellomeres square. Occipital carina joining hypostomal carina behind the mandibles base. Scutellum white (Figure 13c). Metatibia red-black (Figure 13a). Metafemur red and infuscate. Second metasomal tergite quite punctate. Thyridia small (Figure 13d). Metasomal tergites II-V red.

General Distribution: Palearctic.

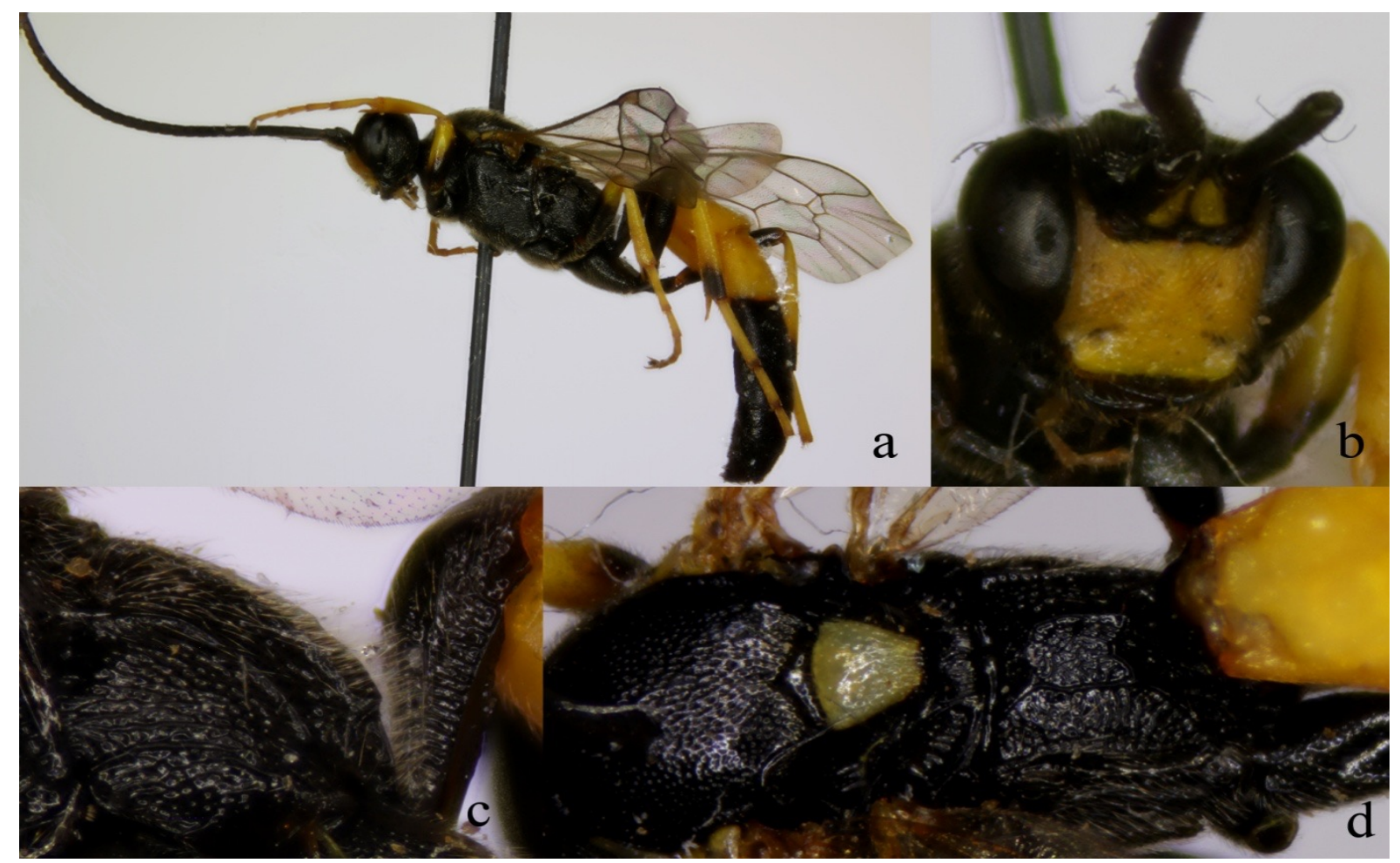

Figure 12. Ichneumon caloscelis a. habitus, lateral view, b. head, facial view, c. metapleuron and propodeum, lateral view and d. mesosoma and first metasomal segment, dorsal view. 


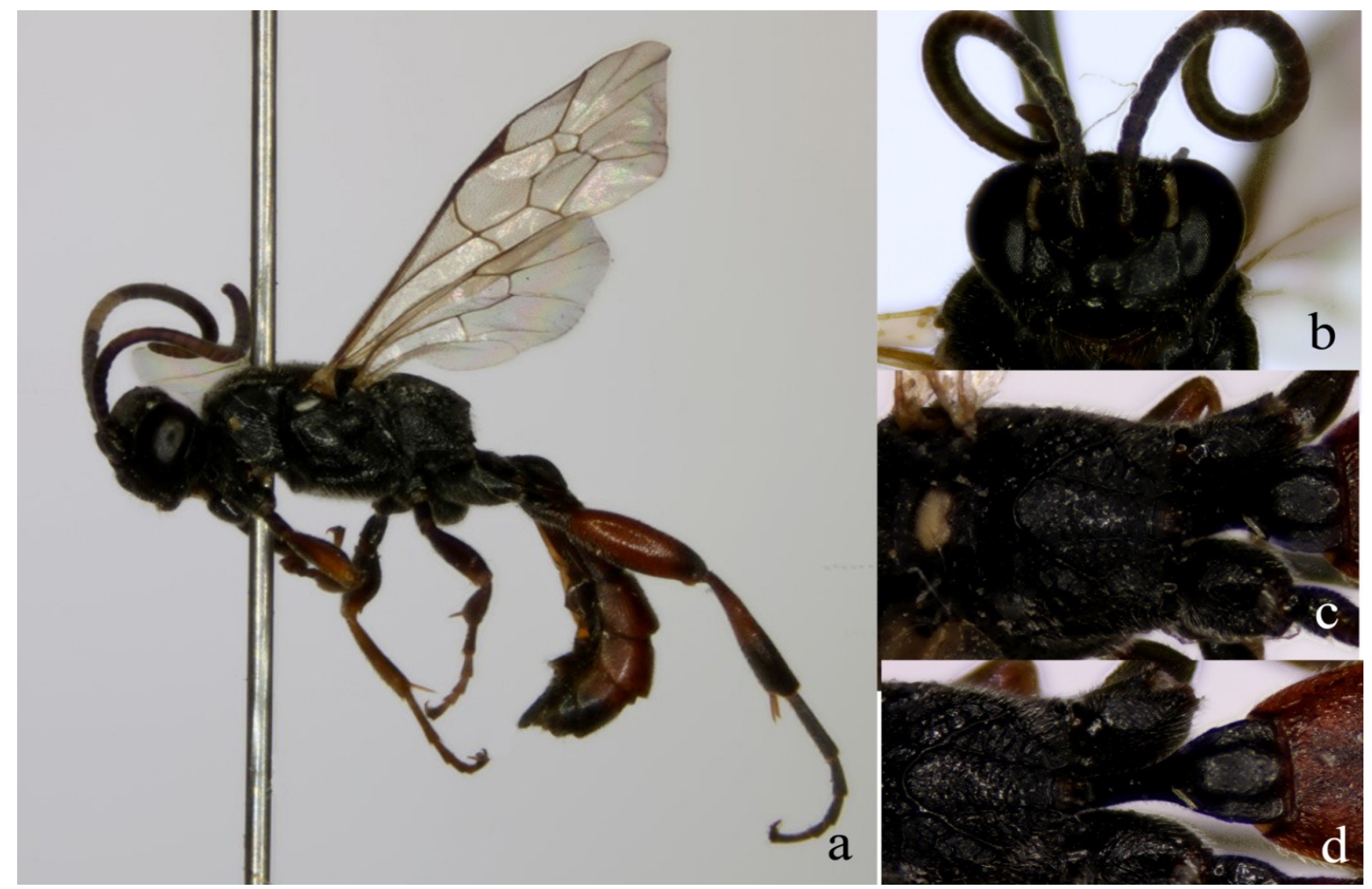

Figure 13. Barichneumon peregrinator a. habitus, lateral view, b. head, facial view c. propodeum, metacoxa, first metasomal tergite, dorsal view and d. propodeum, metacoxa, first and second metasomal tergites and Thyridia of metasoma, dorsal view.

Subfamily Mesochorinae

Mesochorus arenarius (Haliday, 1838)

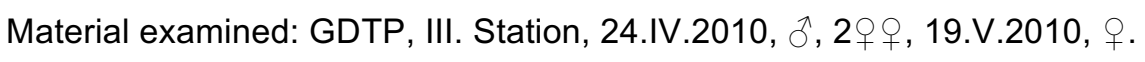

Distribution in Turkey: Turkey (Kolarov, 1989).

Mesochorus fulgurans Curtis, 1833

Material examined: GDTP, III. Station, 13.VI.2010, q.

\section{Subfamily Metopiinae}

\section{Exochus vafer Holmgren, 1873}

Material examined: GDTP, II. Station, 19.V.2010, đ̃.

Distribution in Turkey: Isparta, İzmir (Kolarov et al., 2009); Erzincan (Çoruh \& Kolarov, 2012).

\section{Subfamily Orthocentrinae}

\section{*Symplecis invisitata Rossem, 1981}

Material examined: GDTP, II. Station, 5.XI.2011, ‥

Diagnosis: Labium, labial palpi and maxillary palpi white. Mandibles yellow, teeth of the same length. Eyes strongly converging, malar space very narrow (Figure 14b). Face polished, brown. Pronotum polished, brown. Epomia present. Pleuron 1 brown. Mesopleuron highly polished, speculum smoother and shiny (Figure 14c). Mesoscutum coriaceous, notauli short but well developed anteriorly. Scutellum polished. Forewing without areolet. Nervellus inclivous, intercepted below the middle but discoidella absent. Tegula and wing base white. Front coxae whitish, middle and hind coxae more brown. 
Propodeum with all carina and with rather long widely placed hairs (Figure 14d). Mesopleuron highly polished. First, second, third and fourth metasomal sternit white, with widely placed with hairs, following sternite brown (Figure 14a).

General Distribution: Palearctic, Nearctic.

\section{Subfamily Ophioninae}

Ophion obscuratus Fabricius, 1798

Material examined: GDTP, III. Station, 13.VI.2010, 2 우, ô.

Distribution in Turkey: Erzurum, Muş (Kolarov et al., 2000), Tunceli (Kolarov et al., 2014a), Erzurum (Çoruh \& Çalmaşur, 2016).

\section{Subfamily Pimplinae}

\section{Perithous scurra (Panzer, 1804)}

Material examined: GDTP, II. Station, 1.VI.2012, ․

Distribution in Turkey: Ankara (Kolarov, 1995); Erzurum (Çoruh, 2005).

\section{Pimpla turionellae (Linnaeus, 1758)}

Material examined: GDTP, V. Station, 16.IX.2012, $q$.

Distribution in Turkey: Ankara, Eskişehir, Konya, Nevşehir (Özdemir \& Kılınçer, 1990), Kırklareli (Yurtcan, 2004), Isparta (Gürbüz, 2004), Erzurum (Çoruh, 2005), Osmaniye (Gürbüz et al., 2008), Kasnak Meşesi Tabiatı Koruma Alanı (Kırtay, 2008), Davraz Dağı (Birol, 2010).

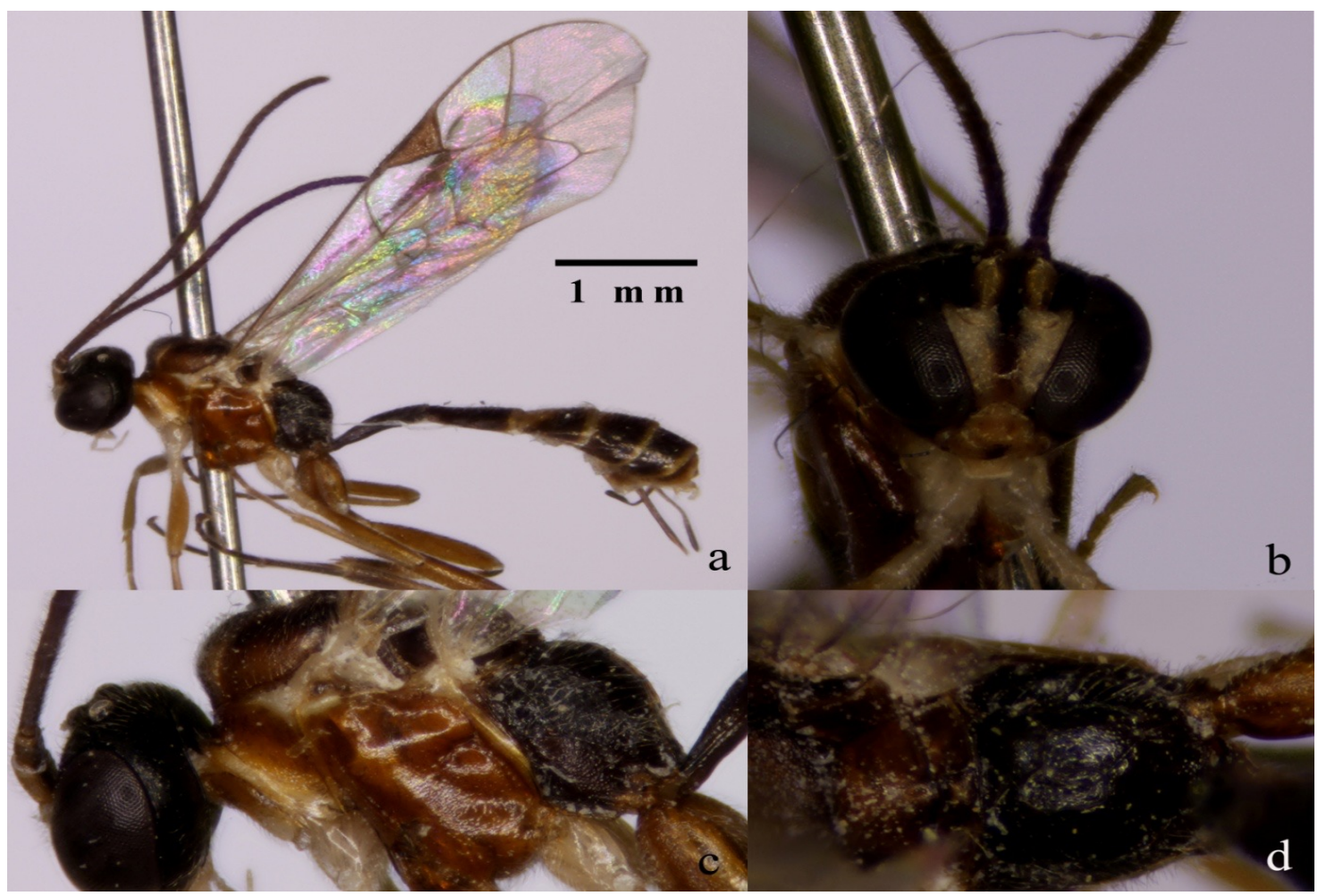

Figure 14. Symplecis invisitata a. habitus, lateral view, b. head, facial view, c. mesosoma, lateral view and d. propodeum and scutellum, dorsal view. 


\section{Clistopyga rufator Holmgren, 1856}

Material examined: GDTP, II. Station, 14.V. 2011, + , 22.V.2011, , IV. Station, 16.VI.2012, $q$.

Distribution in Turkey: Edirne (Yurtcan, 2004), Kırklareli (Yurtcan, 2007), Adana (Buncukçu, 2008), Hatay (Gürbüz et al., 2008).

\section{Iseropus stercorator (Fabricius, 1793)}

Material examined: GDTP, II. Station, 1.VII.2012, 6qq , 12.VI.2012, II. Station, $q, 17 . V I .2012$, ภ̂, 22.VI.2012, ठึ.

Distribution in Turkey: Erzurum (Çoruh \&Özbek, 2008; Çoruh, 2005).

\section{Subfamily Tersilochinae}

Gelanes fusculus Holmgren, 1860

Material examined: GDTP, III. Station, 17.VI.2010, §.

Distribution in Turkey: Habib-i Neccar, Hatay (Gürbüz et al., 2008).

\section{Probles anatolicus Horstmann, 1981}

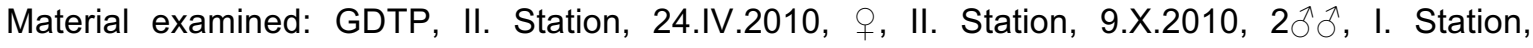
27.V.2012 3우, ô.

Distribution in Turkey: Unknown (Horstmann, 1981).

\section{Subfamily Tryphoninae}

\section{Exenterus abruptorius (Thunberg, 1824)}

Material examined: GDTP, II. Station, 17.VI.2012, 今ึ.

Distribution in Turkey: Konya (Özdemir, 2001).

\section{Monoblastus brachyacanthus (Gmelin, 1790)}

Material examined: GDTP, III. Station, 19.V.2010, ㅇ.

Distribution in Turkey: Erzurum, Tekirdağ (Kolarov \& Beyarslan, 1994), Edirne, Kırklareli (Yurtcan \& Beyarslan, 2002), Isparta, Eğirdir, Uluborlu, Burdur (Gürbüz \& Kolarov, 2006).

\section{Netelia dilatata (Thomson, 1888)}

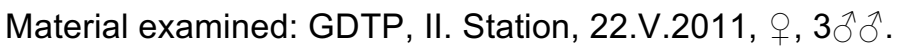

Distribution in Turkey: Erzurum (Kolarov et al., 1999); Ankara, Konya (Özdemir, 2001); Isparta (Gürbüz \& Kolarov, 2006), Elazığ, Eskişehir, Malatya, Sivas (Yaman, 2014), Erzurum (Çoruh \& Kolarov, 2016).

\section{Subfamily Xoridinae}

\section{*Odontocolon quercinus (Thomson,1877)}

Material examined: GDTP, III. Station, 16.IX.2012, q.

Diagnosis: Face black (Figure 15b), mandibles with double teeth. Flagellomere I not longer than flagellomere II. Mesosoma completely black (Figure 15a) and laterally compressed.. Propodeum smooth and shiny. Epomia absent. Metafemur with a large tooth laterally (Figure 15c). Metatibia includes both short and long hairs. Metafemur red color. Pterostigma black-brown. Second metasomal tergite quite spotted (Figure 15d).

Hosts: Cynips quercusfolii Linnaeus 1758 (Hymenoptera:Cyniipidae), Hylotrupes bajulus (Linnaeus 1758) (Coleoptera: Cerambycidae), Monochamus galloprovincialis Oliver (Coleoptera: Cerambycidae).

General Distribution: Western Palearctic, Europe. 


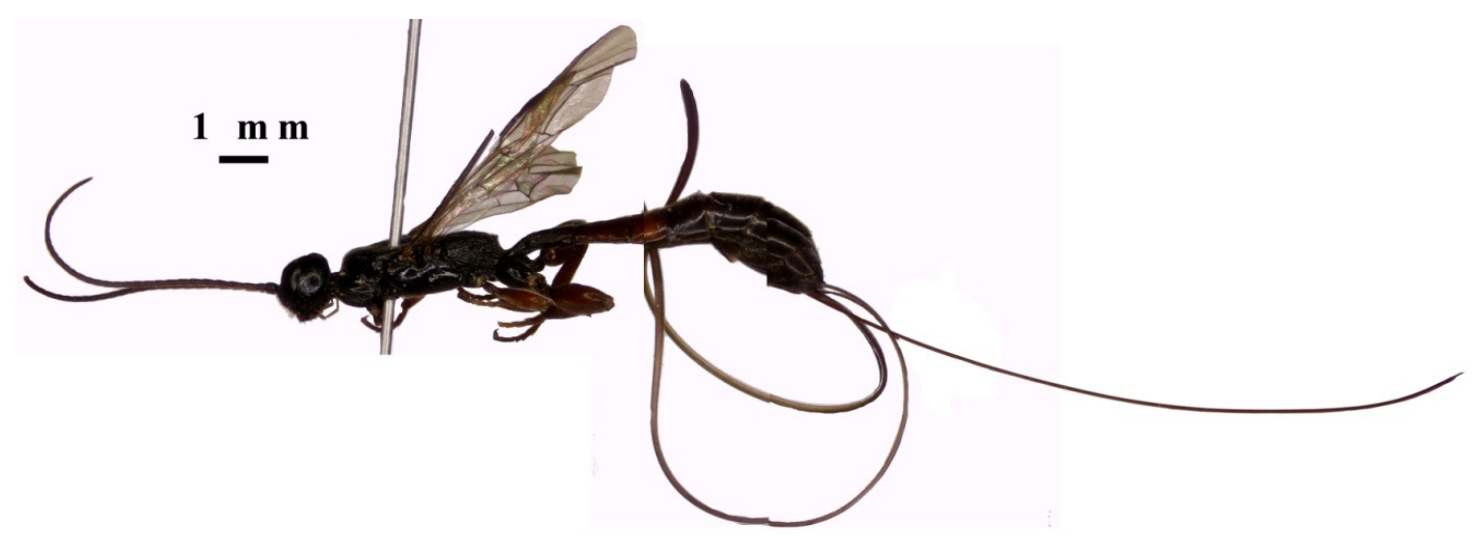

a
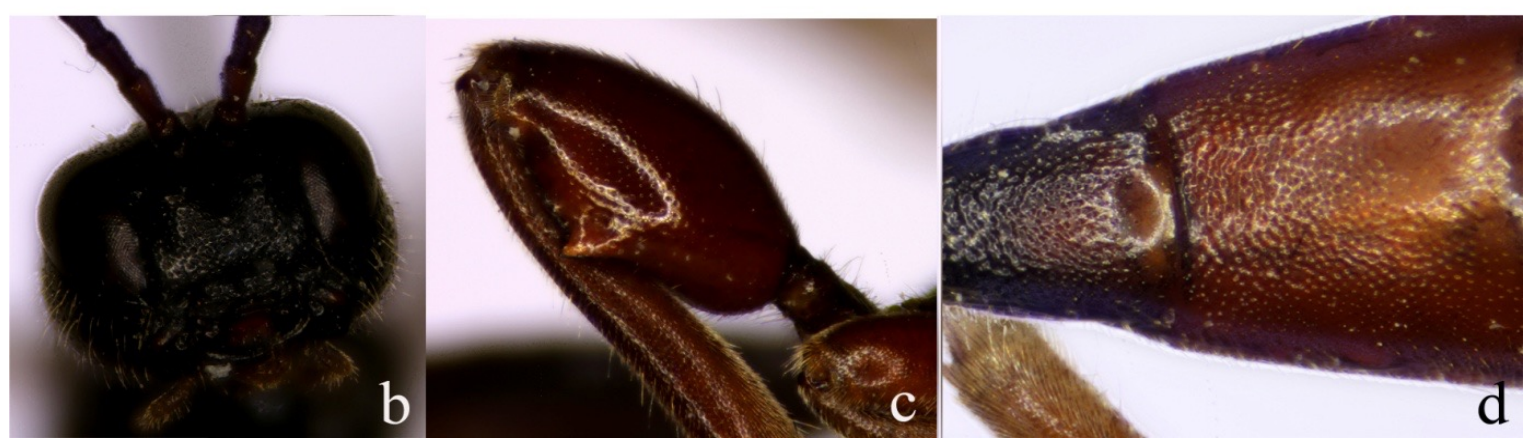

Figure 15. Odontocolon quercinus a. habitus, lateral view, b. head, facial view, c. view of metafemur and d. first and second metasomal tergites, dorsal view.

\section{Acknowledgments}

We gratefully acknowledge the financial support of the project by SDÜ BAP as part of the project No. 2273-D-10. Thanks to Janko Kolarov (University of Plovdiv), Dr. Santiago Bordera (University of Alicante), Dr. Andrey Khalaim (Zoological Institute of Russian Academy of Sciences), Dr. Matthias Riedel, Dr. Valentina Ignatievna Tolkanitz and Ganna Nuzhna for helping to identify species.

\section{References}

Akkaya, A., 2005. Güneydoğu ve Doğu Anadolu Bölgesi'nde Anomaloninae, Banchinae, Collyriinae, Ophioninae ve Pimplinae (Hymenoptera: Ichneumonidae) Türlerinin Sistematik Yönden İncelenmesi. Dicle Üniversitesi Fen Bilimleri Enstitüsü, (Basılmamış) Doktora Tezi, Diyarbakır, $98 \mathrm{~s}$.

Birol, O., 2010. Isparta İli Davraz Dağı Ichneumonidae (Hymenoptera) Faunası Üzerine Bir Araştırma. Süleyman Demirel Üniversitesi, Fen Bilimleri Enstitüsü, (Basılmamış) Yüksek Lisans Tezi, Isparta, $71 \mathrm{~s}$.

Buncukçu, A., 2008. Isparta İli Merkez ve Adana, Yumurtalık İlçesi-Halep Çamlığı Ichneumonidae Türlerinin Tespiti Ve Kültüre Edilebilen Türlerin Biyolojilerinin Araştırılması, Süleyman Demirel Üniversitesi, Fen Bilimleri Enstitüsü, (Basılmamış) Yüksek Lisans, Isparta, 74 s.

Çoruh, S., 2005. Erzurum ve Çevre İllerdeki Pimplinae (Hymenoptera : Ichneumonidae) Türleri Üzerinde Faunistik, Sistematik ve Ekolojik Çalışmalar. Atatürk Üniversitesi, Fen Bilimleri Enstitüsü, (Basılmamış) Doktora tezi, Erzurum, $211 \mathrm{~s}$.

Çoruh, I. \& S. Çoruh, 2008. Ichneumonidae (Hymenoptera) Species Associated with Some Umbelliferae Plants Occurring in Palandöken Mountains of Erzurum, Turkey. Turkish Journal of Zoology, 32, 121-124.

Çoruh, S. \& H. Özbek, 2008. New and rare Ichneumonidae (Hymenoptera) species from Turkey. Zoology in the Middle East, 43: 114-116. 
Çoruh, S. \& J. Kolarov, 2012. Description of the male of Ophion internigrans Kokujev, 1906 (Hymenoptera: Ichneumonidae: Ophioninae) with a key to the Turkish Ophion Fabricius, 1798 Species. Journal Entomological Research Society, 14 (2): 55-60.

Çoruh, S. \& J. Kolarov, 2016. Faunistic notes on the Ichneumonidae (Hymenoptera) of Turkey with a new record. Acta entomologica serbica, 21 (1): inpress.

Çoruh, S. \& Ö. Çalmaşur, 2016. A new and additional records of the Ichneumonidae (Hymenoptera) from Turkey. Turkish Journal of Zoology, 40: 625-629.

Çoruh, S. H. Özbek. \& J. Kolarov, 2004. New and little known Anomaloninae (Hymenoptera, Ichneumonidae) from Turkey. Linzer Biologische Beitrage, 36: 1199-1204.

Çoruh, S., M. F. Gürbüz, J. Kolarov, M. Yurtcan \& A. Buncukçu, 2013. New and little known species of Ichneumonidae (Hymenoptera) for the Turkish Fauna. Journal of the Entomological Research Society, 15: 71-83.

Çoruh, S., J. Kolarov \& İ. Çoruh, 2014a. Ichneumonidae (Hymenoptera) from Anatolia. II. Turkish Journal of Entomology, 38: 279-290.

Çoruh, S., J. Kolarov \& H. Özbek, 2014b. The fauna of Ichneumonidae (Hymenoptera) of eastern Turkey with zoogeographical remarks and host data. Journal of Insect Biodiversity, 2: 1-21.

Çoruh, S., J. Kolarov, İ. Çoruh, 2016. A study of Ichneumonidae (Hymenoptera) from northeastern Anatolia II, with new records. Türkiye Entomoloji Dergisi, 40 (3): 265-280.

Erduran, F. \& U. Cırık, 2011. Gelincik Dağı Tabiat Parkı'nın rekreasyonel peyzaj değerlerinin belirlenmesi. Journal of Agricultural Faculty of Atatürk University, 42 (1): 63-77.

Ghahari, H., M. Fischer \& R. Jussila, 2012. Braconid and ichneumonid wasps (Hymenoptera, Ichneumonoidea) as the parasitoids of Plutella xylostella (L.) (Lepidoptera: Plutellidae) in Iran. Entomofauna, 33 (18): 281-288.

Gürbüz, M. F., 2004. Isparta İli Ichneumonidae (Hymenoptera) Familyası Türleri Üzerine Faunistik ve Sistematik Araştırmalar. Süleyman Demirel Üniversitesi, Fen Bilimleri Enstitüsü, (Basılmamış) Doktora Tezi, Isparta, 68 s.

Gürbüz, M. F. \& J. Kolarov, 2006. A study of Turkish Ichneumonidae (Hymenooptera) II. Tryphoninae. The Gazi Entomological Research Society, 8: 1.

Gürbüz, M. F. \& J. Kolarov, 2008. A study of the Ichneumonidae (Hymenoptera). IV. Cryptinae, Cryptini. Turkish Journal of Zoology, 32: 373-377.

Gürbüz, M. F., T. Ljubomirov, J. Kolarov, M. Yurtcan, M. A. Tabur, S. Çoruh \& A. Buncukçu, 2008. Investigation of the Ichneumonidae, Ampulicidae, Crabronidae and Sphecidae (Hymenoptera, Insect) Fauna in Natural Protection Zones of East Mediteranean Region in Turkey. Tübitak TBAGU/ 168 (106T189) No'lu Proje, 127 s.

Gürbüz, M. F., M. Y. Aksoylar \& A. Buncukçu, 2009a. A faunistic study on Ichneumonidae (Hymenoptera) in Isparta, Turkey. Linzer Biologische Beiträge, 41 (2): 1969-1984.

Gürbüz, M. F., H. Kırtay \& O. Birol, 2009b. A study of Ichneumonidae (Hymenoptera) of Kasnak Oak Forest nature reserve in Turkey with new records. Linzer Biologische Beiträge. 41 (2): 1985-2003.

Horstmann, K., 1981. Revision der Europäischen Tersilochinen II (Hymenoptera, Ichneumonidae). Spixiana Supplement, 4: 1-76.

Kırtay, H., 2008. Isparta Kasnak Meşesi (Quercus vulcanıca Boiss. and Heldr. ex Kotschy) Ormanı Tabiatı Koruma Alanı Ichneumonidae (Hymenoptera) Faunası Üzerine Bir Araştırma. Süleyman Demirel Üniversitesi, Fen Bilimleri Enstitüsü, (Basılmamış) Yüksek Lisans Tezi, Isparta, $77 \mathrm{~s}$.

Kolarov, J., 1989. Taxonomic and faunistic study on Bulgarian Cremastinae (II). Faunistische Abhandlungen, 16 (13): 149-154.

Kolarov, J., 1995. A cataloque of the Turkish Ichneumonidae (Hymenoptera). Entomofauna, Zeitschrift für Entomologie, 7: 137-188.

Kolarov, J. \& A. Beyarslan, 1994. Investigations on the Ichneumonidae (Hym.) fauna of Turkey. 1. Pimplinae and Tryphoninae. Türkiye Entomoloji Dergisi 18 (3): 133-140.

Kolarov, J. \& A. Beyarslan, 1995. "New and little known Turkish Campopleginae (Hymenoptera: Ichneumonidae) 1821". III. National Scientific Conference of Entomology (18-20 September, Sofia, Bulgaria) Proceedings. 
Kolarov, J. \& M. Yurtcan, 2008. A study of the Ichneumonidae (Hymenoptera) of the North Anatolia (Turkey) I. Brachycyrtinae, Cryptinae and Xoridinae. Acta entomologica serbica, 13 (1/2): 89-91.

Kolarov, J., A. Beyarslan \& M. Yurtcan, 1994. "Yeni ve az bilinen Türkiye Anomaloninae türleri (Hymenoptera: Ichneumonidae), 248-251”. XII. Ulusal Biyoloji Kongresi (6-8 Temmuz 1994, Edirne, Türkiye) Bildirileri.

Kolarov, J., H. Özbek \& E. Yıldırım, 1999. New distributional data of the Turkish Ichneumonidae (Hymenoptera). I. Pimplinae and Tryponinae. Journal of the Entomological Research Society, 1 (2): 9-15.

Kolarov, J., S. Pekel, H. Özbek, E. Yıldırım \& Ö. Çalmaşur, 2000. "New distributional data of the Turkish Ichneumonidae (Hymenoptera) III. The subfamily Ophioninae, 349-356”. Türkiye 4. Entomoloji Kongresi (12-15 Eylül 2000, Aydın, Türkiye) Bildirileri.

Kolarov, J., M. Yurtcan \& A. Beyarslan, 2002. "Ichneumonidae species of the Turkish Aegean Region. Parasitic wasps: Evolution, systematics, biodiversity and biological control, 299-305". International Symposium (14-17 May 2001, Agroinform, Koszeg-Hungary) Proceedings.

Kolarov, J., S. Çoruh, M. Yurtcan \& M. F. Gürbüz, 2009. A study of Metopiinae from Turkey with description of a new species (Hymenoptera: Ichneumonidae). Zoology İn The Middle East, 46: 75-82.

Kolarov, J., E. Yıldırım, S. Çoruh \& M. Yüksel, 2014a. Contribution to the knowledge of the Ichneumonidae (Hymenoptera) fauna of Turkey. Zoology in the Middle East, 60: 154-161.

Kolarov, J., S. Çoruh \& I. Çoruh, 2014b. Ichneumonidae (Hymenoptera) from Anatolia. III. Turkish Journal of Entomology, 38: 377-388.

Kolarov, J., S. Çoruh \& İ. Çoruh, 2016. Contribution to the knowledge of the Ichneumonidae (Hymenoptera) fauna of Turkey from northeastern Anatolia, Part I. Turkish Journal of Zoology, 40: 40-56.

Kopylov, D. S. \& H. Zhang, 2015. New ichneumonids (Insecta: Hymenoptera: Ichneumonidae) from the Lower Cretaceous of north China. Cretaceous Research, 52: 591-604.

Okyar, Z, M. Yurtcan, A. Beyarslan \& N. Aktaş, 2012. The Parasitoid complex of White-spotted Pinion Cosmia diffinis (Linnaeus, 1767) (Lepidoptera: Noctuidae) on Ulmus minor Miller (Ulmaceae) in Edirne Province (European Turkey). Journal of the Kansas Entomological Society, 85 (2): 91-96.

Özbek, H., S. Pekel \& J. Kolarov, 2000. New distributional data of the Turkish Ichneumonidae (Hymenoptera) II. Ctenopelmatinae and Campopleginae. Journal of the Entomological Research Society, 2: 17-24.

Özdemir, Y., 1996. İç Anadolu Bölgesinde tespit edilen Banchinae ve Ichneumoninae (Hymenoptera: Ichneumonidae) türleri. Bitki Koruma Bülteni, 36 (3-4): 91-104.

Özdemir, Y., 2001. İç Anadolu Bölgesinde saptanan Diplazontinae ve Tryphoninae (Hymenoptera: Ichneumonidae) türleri. Türkiye Entomoloji Dergisi, 25 (3): 183-191.

Özdemir, Y. \& N. Kılınçer, 1990. "İç Anadolu Bölgesinde saptanan Pimplinae ve Ophioninae (Hymenoptera: Ichneumonidae) türleri, 309-318”. Türkiye II. Biyolojik Mücadele Kongresi (26-29 Eylül 1990, Ankara, Türkiye) Bildirileri.

Özdemir, Y. \& Güler Y., 2009. Sultandağı Havzası kiraz bahçelerinde tespit edilen ichneumonidae (Hymenoptera) türleri. Bitki Koruma Bülteni, 49 (3): 135-143.

Pekel, S. \& H. Özbek, 2000. Erzurum ili Cremastinae (Hymenoptera: Ichneumonidae) altfamilyası üzerinde faunistik ve sistematik bir çalışma. Türkiye Entomoloji Dergisi, 24 (3): 215-228.

Riedel, M., S. Çoruh \& H. Özbek, 2010. Contribution to the Ichneumoninae Hymenoptera, Ichneumonidae) fauna of Turkey, with description of three new species. Türkiye Entomoloji Dergisi, 34 (2): 133-156.

Sedivy, J., 1959. Wissenschaftliche Ergebnisse der zoologischen Expedition des National-Museums in Prag nach der Tuerkei. 26. Hymenoptera, Ichneumonidae. Acta Faunistica Entomologica Musei Nationalis Pragae, 33: $107-116$.

Townes, H. K., 1969. The genera of Ichneumonidae: 1. Ephialtinae to Agriotypinae. Memoirs of the American Entomological Institute, 11: 1-300.

Wahl, D. B. \& M. J. Sharkey, 1993. "Superfamily Ichneumonoidae, 358-449". In: The Hymenoptera of the World, An Identification Guide to Families (Eds. H. Goulet \& J. Huber), Canada Agriculture, Canada, 668 pp.

Yaman, G., 2014. Türkiye Tryphoninae (Hymenoptera: Ichneumonidae) Türlerinin Kontrol Listesi. Trakya Üniversitesi, Fen Bilimleri Enstitüsü, (Basılmamış) Yüksek Lisanas Tezi, Edirne, 88 s. 
Yu, D., C. Van Achterberg \& K. Horstmann, 2012. Taxapad 2012, Ichneumonoidae 2011. Database on flash-drive. www.taxapad.com, Ottawa, Ontario, Canada.

Yurtcan, M., 2004. Trakya Bölgesi Pimplinae (Hymenoptera: Ichneumonidae) Faunasının Taksonomik ve Faunistik Yönden Araştırılması. Trakya Üniversitesi Fen Bilimleri Enstitüsü (Basılmamış) Doktora Tezi, Edirne, $110 \mathrm{~s}$.

Yurtcan, M., 2007. Ephialtini tribe (Hymenoptera, Ichneumonidae, Pimplinae) of Turkish Thrace region, Entomofauna, 28: 389-404.

Yurtcan, M., A. Beyarslan \& J. Kolarov, 1999. Investigations on the Ichneumonidae (Hymenoptera) fauna of Turkey. V. Diplazontinae and Ichneumoninae. Acta Zoologica Bulgarica, 1: 36.

Yurtcan, M. \& A. Beyarslan, 2002. The species of Tryphoninae (Hymenoptera: Ichneumonidae) in Turkish Thrace. Turkish Journal of Zoology, 26: 77-95. 